\title{
Editorial
}

\section{Healthcare Sensors for Daily Life}

\author{
Toshiyo Tamura, ${ }^{1}$ Wenxi Chen, ${ }^{2}$ Kwang-Suk Park, ${ }^{3}$ and Rita Paradiso ${ }^{4}$ \\ ${ }^{1}$ Faculty of Biomedical Engineering, Osaka Electro-Communication University, Neyagawa, Osaka 572-8530, Japan \\ ${ }^{2}$ Biomedical Information Technology Laboratory, The University of Aizu, Aizu-Wakamatsu, Fukushima 965-8580, Japan \\ ${ }^{3}$ Department of Biomedical Engineering, College of Medicine, Seoul National University, Seoul 110-799, Republic of Korea \\ ${ }^{4}$ Smartex srl, Navacchio, 56023 Pisa, Italy
}

Correspondence should be addressed to Toshiyo Tamura; tamurat@isc.osakac.ac.jp

Received 11 October 2015; Accepted 11 October 2015

Copyright (C) 2016 Toshiyo Tamura et al. This is an open access article distributed under the Creative Commons Attribution License, which permits unrestricted use, distribution, and reproduction in any medium, provided the original work is properly cited.

With the advent of information and communication technology (ICT) and its pervasive application in medical and healthcare domains, life expectancy worldwide has extended over $80 \mathrm{~s}$ in both females and males. Decline in health condition due to aging would deteriorate personal quality of life and pose extreme burden in global financial expenditure. Healthy aging is therefore of paramount importance.

To support "aging in place" and "daily health promotion," accurate detection and early warning of health condition change are indispensable and imminent. Numerous technical innovations are provoked. Seamless monitoring of physiological information in various living scenarios without disturbing daily activities over a long-term period can significantly enhance caregivers' ability to deliver evidence-based care and becomes one of the effective approaches.

In pursuing seamless monitoring of multifaceted physiological information in daily life environment, many academic goals have been challenged and variety of technological conundrums has been experienced over the past decades. Endeavors in research and development have gone through different approaches which enhance several aspects such as miniaturization, comfortableness, and concealment to achieve better user affinity in different application scenarios of daily life. Miniaturization aims at implementation of portable monitors for ambulatory application. Wearable monitors target for pervasive application in daily activities without much discomfort. Invisible methods are usually realized by concealing sensors or transducers into furniture and appliances for indoor application. Some of these outcomes have matured and have been commercialized in daily setting; yet some of them remain unanswered and require further elaboration.

This special issue focuses on device development, data analysis, and system integration aiming at health monitoring in daily life which has considerable potential for preventing and predicting diseases, without significant discomfort or inconvenience to the home users. Thus, this issue contains (1) review of the devices used for falling risk assessment; (2) discussion of long-term data collection and analysis using Big Data techniques; (3) new devices for further application of new technologies.

The problem in elderly community is the fall risk assessment in daily life environment. There are several inertial sensors used to provide the fall risk assessment and prevention. Image processing technology also has been applied in the home. In this issue, the image processing with multisensor fusion technologies to assess and detect the falling is reviewed.

Secondly, long-term records collected from daily living need to be evaluated and help for further prevention and prediction of diseases. In the issue, cardiac recovery process in the pregnancy and after delivery was analyzed from the long-term pulse records.

Thirdly, three papers present new ideas for monitoring at daily living. The wearable deep body thermometer may be a promising technology for preventing heat stroke and monitoring mental stress and circadian rhythms.

Other papers also show technologies to improve current healthcare sensors. For improving quality of life, the elderly used to wear pedometer. The unique algorithm is applied to 
reduce power consumption of pedometer. One more paper presents the cardiovascular monitor for real time detection of atrial fibrillation.

\title{
Acknowledgments
}

Finally, we would like to thank all the authors for their valuable contributions and also the reviewers for their critical help necessary to achieve a high level of papers' quality and make the completion of this special issue possible. Also, we would like to thank all members of the editorial board for approving this special issue.

\author{
Toshiyo Tamura \\ Wenxi Chen \\ Kwang-Suk Park \\ Rita Paradiso
}




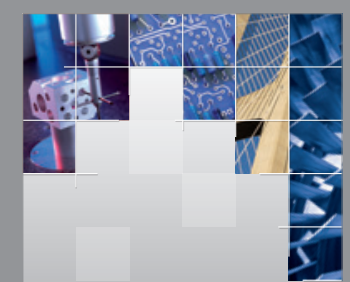

\section{Enfincering}
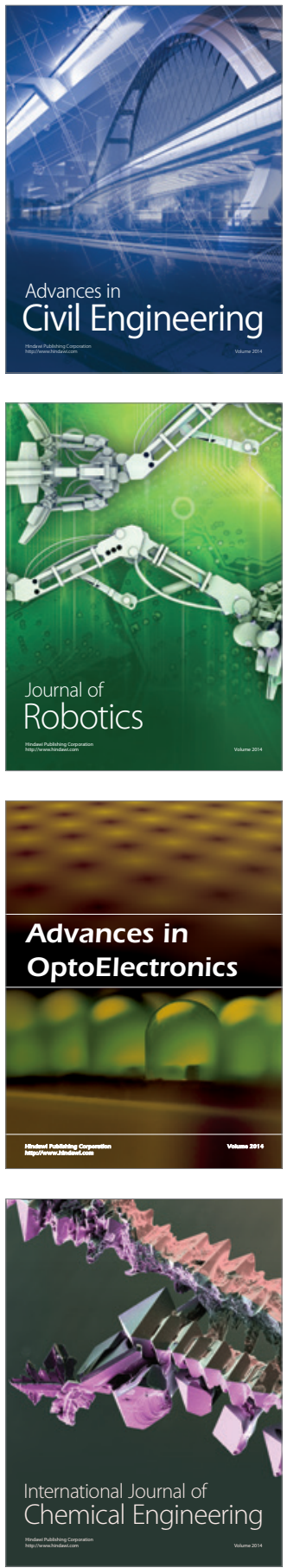

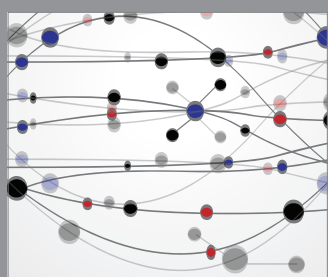

The Scientific World Journal

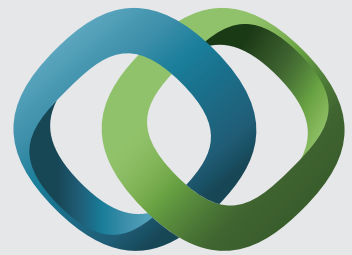

\section{Hindawi}

Submit your manuscripts at

http://www.hindawi.com
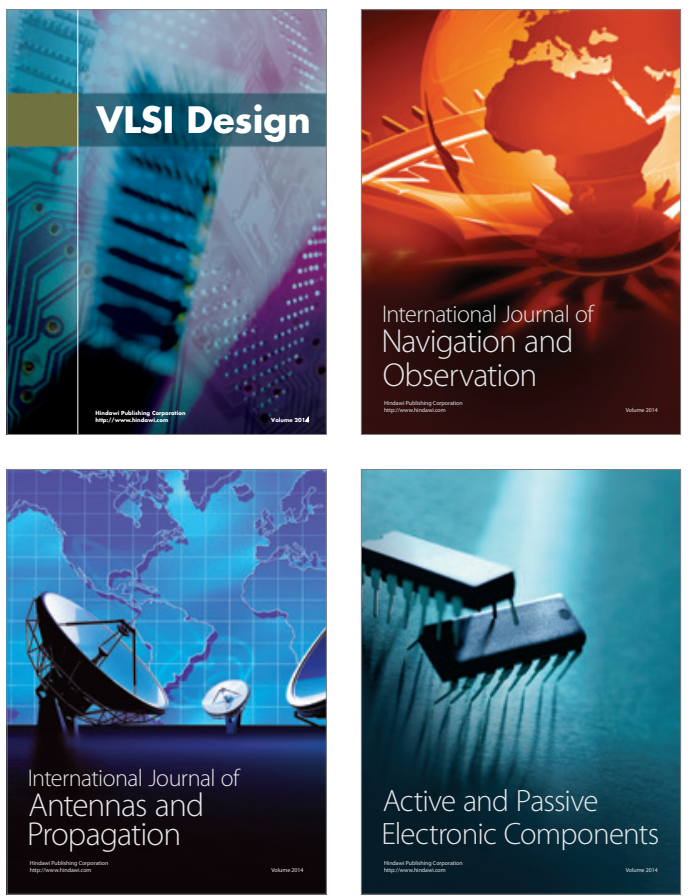
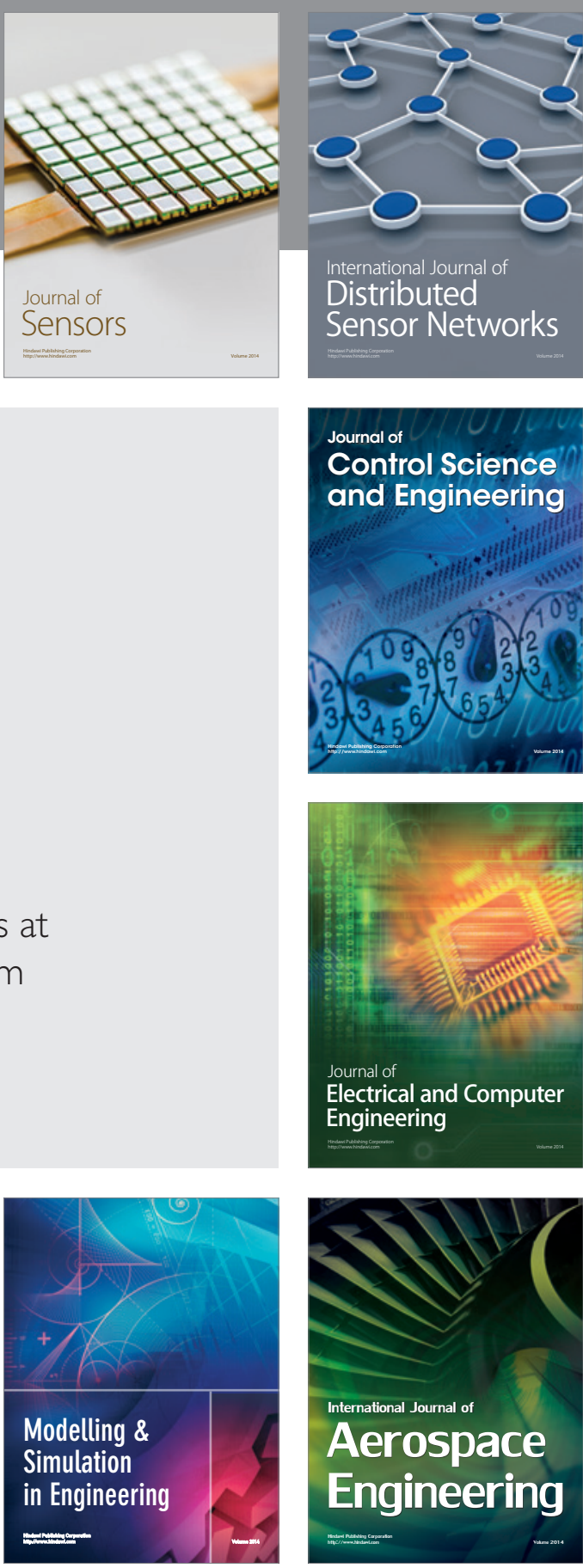

International Journal of

Distributed

Sensor Networks

Journal of

Control Science

and Engineering
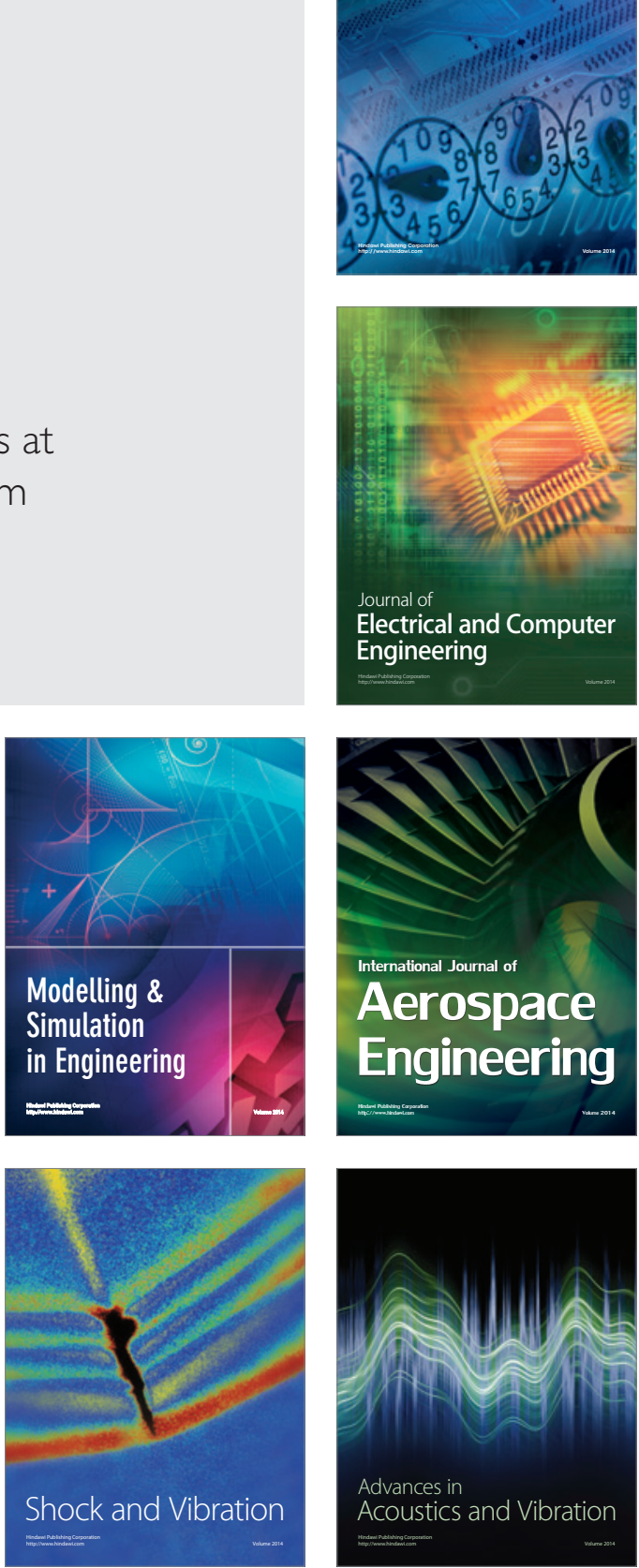